\title{
EDITORIAL
}

\section{Thoracic malignancies, cisplatin and renal function}

\author{
J. Nortier* and J-P. Sculier ${ }^{\#}$
}

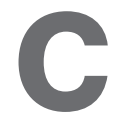
isplatin (cis-dichlorodiammine-platinum II or CDDP) is the central drug used in the management of thoracic malignancies. The first trials were published in the 1970s [1], showing activity in lung cancer. Since then it has been shown to improve survival in advanced nonsmall cell lung cancer (NSCLC) [2] and survival and cure in combination with radiotherapy in locoregional NSCLC and prior to [3] or after resection in early NSCLC [4]. It is also a key drug in association with etoposide $[5,6]$ in the treatment of small cell lung cancer (SCLC), where it significantly improves survival. In mesothelioma, it has been shown to be the most active drug $[7,8]$.

The toxicity profile of cisplatin is unique. Dose-limiting toxicity is not haematological as with cytotoxic chemotherapy, but renal with the development of acute renal failure. The administration of higher doses of cisplatin $\left(100-120 \mathrm{mg} \cdot \mathrm{m}^{-2}\right)$ with a better therapeutic index has been made possible by mannitol-induced diuresis and hyperhydration [9]. Nevertheless, the use of such dosages is associated with chronic toxicity with not only renal but also auditive neurological impairments, preventing long-term treatment as shown in a phase II randomised trial [10]. In this study, conducted by the European Lung Cancer Working Party (ELCWP) in patients with advanced NSCLC, high-dose cisplatin $\left(120 \mathrm{mg} \cdot \mathrm{m}^{-2}\right)$ was compared to moderate-dose cisplatin $\left(60 \mathrm{mg} \cdot \mathrm{m}^{-2}\right)$ plus carboplatin $\left(200 \mathrm{mg} \cdot \mathrm{m}^{-2}\right)$ [10]. High-dose cisplatin was significantly associated with more renal ( $36 \%$ versus $19 \%)$, auditive (16\% versus $4 \%$ ) and neurological (16\% versus $0 \%$ ) toxicity without significantly different response rates ( $21 \%$ versus $23 \%)$.

Various attempts have been made in order to reduce or avoid the renal toxicity of cisplatin without reducing its effectiveness. Carboplatin, another platinum derivative with reduced nephrotoxicity, has been proposed as a substitute of cisplatin. Comparison of the two drugs in NSCLC has been the topic of 10 published randomised trials and three meta-analyses summarised in a recent review [2]. In randomised trials, the trend was in favour of cisplatin, both in terms of response and survival. The meta-analyses confirmed this impression; the results were statistically significant in favour of cisplatin if the analysis was restricted to the regimens using new drugs combined with platinum derivatives. Thus, cisplatin should be preferred to carboplatin because of a better survival effect.

\footnotetext{
*Dept of Nephrology, Hôpital Erasme, Université Libre de Bruxelles. ${ }^{\#}$ Dept of Intensive Care and Thoracic Oncology, Institut Jules Bordet, Centre des Tumeurs de I'Université Libre Bruxelles, Brussels, Belgium.

CORRESPONDENCE: J-P. Sculier, Dept of Critical Care and Thoracic Oncology, Institut Jules Bordet, Université Libre Bruxelles, 1 rue Héger-Bordet, B-1000 Brussels, Belgium. E-mail: sculier@bordet.be
}

Data are too limited to propose this approach for SCLC and mesothelioma. A second approach to increase platinum doses is to combine cisplatin with carboplatin, two drugs with different toxicity profiles. The theoretical advantage of such a regimen is to allow administration of higher dose-intensity of platinum derivatives than low-dose cisplatin alone. This hypothesis was also tested by the ELCWP [11] in a phase III randomised trial conducted in patients with metastatic NSCLC. In association with mitomycin $\left(6 \mathrm{mg} \cdot \mathrm{m}^{-2}\right)$ and ifosfamide $\left(3 \mathrm{~g} \cdot \mathrm{m}^{-2}\right)$, the combination of moderate dosages of cisplatin $\left(60 \mathrm{mg} \cdot \mathrm{m}^{-2}\right)$ and carboplatin $\left(200 \mathrm{mg} \cdot \mathrm{m}^{-2}\right)$, CarboMIP regimen, was compared with cisplatin $\left(50 \mathrm{mg} \cdot \mathrm{m}^{-2}\right)$ alone, MIP regimen,. The study failed to demonstrate a significant improvement in response or survival but toxicity, mainly haematological, was higher with the CarboMIP regimen. A third method is the use of so-called low dosages of cisplatin. Five randomised trials have been performed on this [2]. None were able to report a significant advantage in favour of high dosages of cisplatin $\left(100-120 \mathrm{mg} \cdot \mathrm{m}^{-2}\right)$ in comparison to lower dosages $\left(50-60 \mathrm{mg} \cdot \mathrm{m}^{-2}\right)$.

All these studies were based on the assessment of renal function by creatinine clearance. Indeed, the determination of serum creatinine level is not sufficiently sensitive to evaluate the renal function at initiation or during any chemotherapeutic treatment. The determination of the glomerular filtration rate is mandatory. This parameter is reflected by the creatinine clearance measured from $24 \mathrm{~h}$ urine collection or calculated using the Cockroft-Gault formula or the abbreviated Modification of Diet in Renal Disease formula [12]. This is particularly helpful in elderly patients with pre-existing chronic kidney disease (CKD), most often associated to cardiovascular comorbidities such as diabetes, ischaemic heart disease and/or hypertension. In these complex clinical situations, pharmacokinetic profiles of many drugs are disturbed and dosage adaptations remain difficult in daily practice. Moreover, pre-renal failure due to extracellular fluid depletion (poor intake, vomiting, diarrhoea, sepsis, etc.) is also commonly observed. Drugs themselves may trigger or worsen hypovolemia, e.g. non-steroidal anti-inflammatory drugs, by vasoconstricting afferent glomerular arterioles.

The prevalence of CKD in cancer patients is not well-defined. In a French retrospective study performed on a group of 4,684 cancer patients, LAUNAY-VACHER et al. [13] found that almost three fifths of patients (57.4\%) suffered from a mild-to-moderate renal insufficiency (defined as a reduced glomerular filtration rate between 60 and $90 \mathrm{~mL} \cdot \mathrm{min}^{-1} \cdot 1.73 \mathrm{~m}^{-2}$ ), although only $7.2 \%$ exhibited an increase in serum creatinine $\left(>1.1 \mathrm{mg} \cdot \mathrm{dL}^{-1}\right)$. Of the 7,181 anticancer drug prescriptions, $53.4 \%$ required dosage adjustments for renal insufficiency. Of the patients treated, $80 \%$ 
received at least one such drug. The authors concluded that CKD was quite common in patients with cancer, and that drug dosage adjustments were often necessary.

In this issue of European Respiratory Journal, MÁTHÉ et al. [14] retrospectively evaluated the impact of cardiovascular comorbidities on cisplatin nephrotoxicity in patients over the first to fourth cycles of cisplatin. These investigators show that cisplatin-induced nephrotoxicity was significantly enhanced in the case of underlying hypertension and ischaemic heart disease, even more so if diabetes was also involved. Taking into account that in most kidney diseases long-term outcome is determined by the severity of the tubulo-interstitial damage, it appears crucial to provide the clinician with accurate tools for monitoring the extent of tubulo-interstitial lesions prior to and during any chemotherapy. Urinary biomarkers have been developed for several years and should become more and more attractive in the very near future, for at least three reasons [15, 16]. 1) Being well categorised by nephron segment they are able to specifically and quantitatively reflect any suspected insult. 2) Their longitudinal monitoring should predict progression of tubulointerstitial lesions. 3) The impact of therapeutic strategies on their urinary excretion rate would be directly related to any prognosis of kidney function. Besides the well-known urinary excretion of low-molecular weight ("tubular") proteins, more recent urinary biomarkers demonstrated potential value in the management of CKD but also in unmasking early transient acute kidney injury. For example, kidney injury molecule- 1 is a transmembrane glycoprotein upregulated in dedifferentiated proximal tubule epithelial cells and shed in urine following acute injury [17]. Urinary neutrophil gelatinase-associated lipocalin is not only associated with loss of kidney function but is also one of the earliest proteins induced in the kidney after an ischaemic or toxic insult [18]. Its long-term monitoring should help in measuring the response to nephroprotective treatment. Associating the measurement of albuminuria and urinary $\mathrm{N}$ acetyl- $\beta$-glucosaminidase is suggested by some authors in an attempt to better predict diabetic nephropathy [19]. It is important to note that long-term studies will be necessary to validate specific biomarkers panels in CKD patient populations, particularly in the field of renal inflammation and/or fibrosis.

In conclusion, the renal management of cancer patients is a key process with many potential developments in the very near future. Characterisation and validation of highly sensitive and specific urinary biomarkers should optimise our understanding of the pathophysiology of renal diseases and our capacity to predict any earlier kidney injury. Furthermore, it would enhance the development of more targeted interventions. By minimising nephrotoxic insults induced by chemotherapeutic agents, these measures should hopefully improve the survival rate of cancer patients.

\section{STATEMENT OF INTEREST}

None declared.

\section{REFERENCES}

1 Rossof AH, Bearden JD III, Coltman CA Jr. Phase II evaluation of cis-diamminedichloroplatinum(II) in lung cancer. Cancer Treat Rep 1976; 60: 1679-1680.
2 Sculier JP, Moro-Sibilot D. First- and second-line therapy for advanced nonsmall cell lung cancer. Eur Respir J 2009; 33: 915-930.

3 Auperin A, Le PC, Pignon JP, et al. Concomitant radio-chemotherapy based on platin compounds in patients with locally advanced non-small cell lung cancer (NSCLC): a meta-analysis of individual data from 1764 patients. Ann Oncol 2006; 17: 473-483.

4 Berghmans T, Paesmans M, Meert AP, et al. Survival improvement in resectable non-small cell lung cancer with (neo)adjuvant chemotherapy: results of a meta-analysis of the literature. Lung Cancer 2005; 49: 13-23.

5 Mascaux C, Paesmans M, Berghmans T, et al. A systematic review of the role of etoposide and cisplatin in the chemotherapy of small cell lung cancer with methodology assessment and meta-analysis. Lung Cancer 2000; 30: 23-36.

6 Pujol JL, Carestia L, Daures JP. Is there a case for cisplatin in the treatment of small-cell lung cancer? A meta-analysis of randomized trials of a cisplatin-containing regimen versus a regimen without this alkylating agent. Br J Cancer 2000; 83: 8-15.

7 Berghmans T, Paesmans M, Lalami Y, et al. Activity of chemotherapy and immunotherapy on malignant mesothelioma: a systematic review of the literature with meta-analysis. Lung Cancer 2002; 38: 111-121.

8 Scherpereel A, Astoul P, Baas P, et al. Guidelines of the European Respiratory Society and the European Society of Thoracic Surgeons for the management of malignant pleural mesothelioma. Eur Respir J 2010; 35: 479-495.

9 Hayes DM, Cvitkovic E, Golbey RB, et al. High dose cis-platinum diammine dichloride: amelioration of renal toxicity by mannitol diuresis. Cancer 1977; 39: 1372-1381.

10 Sculier JP, Klastersky J, Giner V, et al. Phase II randomized trial comparing high-dose cisplatin with moderate- dose cisplatin and carboplatin in patients with advanced non-small-cell lung cancer. European Lung Cancer Working Party. J Clin Oncol 1994; 12: 353-359.

11 Sculier JP, Lafitte JJ, Paesmans M, et al. Phase III randomized trial comparing moderate-dose cisplatin to combined cisplatin and carboplatin in addition to mitomycin and ifosfamide in patients with stage IV non-small-cell lung cancer. Br J Cancer 2000; 83: 1128-1135.

12 National Kidney Foundation. K/DOQI clinical practice guidelines for chronic kidney disease: evaluation, classification, and stratification. Am J Kidney Dis 2002; 39: Suppl. 1, S1-S266.

13 Launay-Vacher V, Oudard S, Janus N, et al. Prevalence of renal insufficiency in cancer patients and implications for anticancer drug management: the renal insufficiency and anticancer medications (IRMA) study. Cancer 2007; 110: 1376-1384.

14 Máthé $\mathrm{C}$, Bohács $\mathrm{A}$, Duffek $\mathrm{L}$, et al. Cisplatin nephrotoxicity aggravated by cardiovascular disease and diabetes in lung cancer patients. Eur Respir J 2011; 37: 888-894.

15 Ferguson MA, Vaidya VS, Bonventre JV. Biomarkers of nephrotoxic acute kidney injury. Toxicology 2008; 245: 182-193.

16 Waanders F, Navis G, van GH. Urinary tubular biomarkers of kidney damage: potential value in clinical practice. Am J Kidney Dis 2010; 55: 813-816.

17 Perico N, Cattaneo D, Remuzzi G. Kidney injury molecule 1: in search of biomarkers of chronic tubulointerstitial damage and disease progression. Am J Kidney Dis 2009; 53: 1-4.

18 Han WK, Wagener G, Zhu Y, et al. Urinary biomarkers in the early detection of acute kidney injury after cardiac surgery. Clin J Am Soc Nephrol 2009; 4: 873-882.

19 Kern EF, Erhard P, Sun W, et al. Early urinary markers of diabetic kidney disease: a nested case-control study from the Diabetes Control and Complications Trial (DCCT). Am J Kidney Dis 2010; 55: 824-834. 